\title{
Impulsive Control for Synchronization of Lorenz Chaotic System
}

\section{Wenxiang Zhang, Zhanji Gui, Kaihua Wang*}

School of Mathematics and Statistics, Hainan Normal University, Haikou, Hainan, China.

Email: *kaihuawang@qq.com

Received 2012

\begin{abstract}
Chaotic synchronization is the key technology of secure communication. In this paper, an impulsive control method for chaotic synchronization of two coupled Lorenz chaotic system was proposed. The global asymptotic synchronization of two Lorenz systems was realized by using the linear error feedback of the state variables of the drive system and the response system as impulsive control signal. Based on stability theory of impulsive differential equation, conditions were obtained to guarantee the global asymptotic synchronization of two Lorenz systems. The theory analysis and computer simulation results validated its effectiveness.
\end{abstract}

Keywords: Lorenz System; Impulsive Control; Synchronization

\section{Introduction}

As the key technology of secure communication, chaotic synchronization has been widely development since Pecora and Carroll [1] proposed the principle of chaos synchronization and realized it in the circuit in 1990. Several synchronization methods have been proposed so far, such as drive-response synchronization, coupling synchronization, feedback-perturb synchronization, self-adapt synchronization, impulse synchronization, and so on [2-4]. Impulse synchronization has been widely appreciated by researcher and made some good progress [5-8]. However, many of the impulsive control methods for synchronization are subject to certain restrictions. Paper [9] studied impulsive synchronization for Rössler chaotic system, and paper [10] researched impulsive control for synchronization of a class of chaotic system. In their papers the drive signal is generated by the impulsive signal and continuous signal of the system variables, so the controller is very complicated. In this paper, we use an impulsive control method, and design the controller for Lorenz chaotic system. Designed controller is simple and easy to be realized.

\section{Problem Formulation}

In this section, we study the impulsive control of Lorenz chaotic system [11] described by the following differential equation:

*Corresponding author.

$$
\left\{\begin{array}{l}
\dot{x}_{1}=-\sigma x_{1}+\sigma x_{2} \\
\dot{x}_{2}=\rho x_{1}-x_{2}-x_{1} x_{3} \\
\dot{x}_{3}=-\beta x_{3}+x_{1} x_{2}
\end{array}\right.
$$

where $\sigma, \rho, \beta$ are system's positive real number parameters. We choose the parameters $\sigma=10, \rho=28$, $\beta=8 / 3$, the initial condition is given by

$\left(x_{1}(0), x_{2}(0), x_{3}(0)\right)^{T}=(0,0,1)^{T}$. The uncontrolled trajectories are shown in Figure 1, which is the notable Lorenz attractor.

System (1) can be rewritten into the following form:

$$
\dot{x}=A x+g(x)
$$

where $x=\left(x_{1}, x_{2}, x_{3}\right)^{T}$ is state variable,

$$
A=\left(\begin{array}{ccc}
-\sigma & \sigma & 0 \\
\rho & -1 & 0 \\
0 & 0 & -\beta
\end{array}\right), \quad g(x)=\left(\begin{array}{c}
0 \\
-x_{1} x_{3} \\
x_{1} x_{2}
\end{array}\right) .
$$

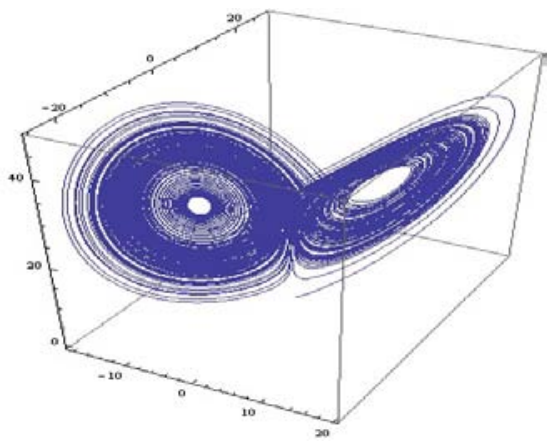

Figure 1. Lorenz attractor. 
Regarding (2) as a drive system, the response system can be described as:

$$
\dot{y}=A y+g(y)
$$

where $y=\left(y_{1}, y_{2}, y_{3}\right)^{T}$ is state variable of response system. Using linear feedback of synchronization error as impulsive signal, we can obtain the following impulsive response system:

$$
\left\{\begin{array}{l}
\dot{y}=A y+g(y) \quad t \neq t_{k}, k=1,2, \cdots \\
\Delta y=B_{k}(y-x) \quad t=t_{k} \\
y\left(t_{0}^{+}\right)=y_{0}
\end{array}\right.
$$

where $B_{k} \in R^{3 \times 3}(k=1,2, \cdots)$ are constant matrices describing the linear nature of the driving impulses, $\Delta y \square y\left(t_{k}^{+}-t_{k}^{-}\right)$, $y\left(t_{k}^{-}\right) \square y\left(t_{k}\right)$.

From system (2) and system (4), the error dynamics is given as following:

$$
\left\{\begin{array}{l}
\dot{e}=A e+M(x, y) g(e) \quad t \neq t_{k}, k=1,2, \cdots \\
\Delta e=B_{k} e \quad t=t_{k} \\
e\left(t_{0}^{+}\right)=e_{0}
\end{array}\right.
$$

here $e=y-x=\left(y_{1}-x_{1}, y_{2}-x_{2}, y_{3}-x_{3}\right)^{T}$ is synchronization error,

$$
M(x, y)=\left(\begin{array}{ccc}
0 & 0 & 0 \\
-y_{3} & 0 & -x_{1} \\
y_{2} & x_{1} & 0
\end{array}\right)
$$

\section{Synchronization of Lorenz Chaotic System}

Theorem: Denote $\beta_{k}, \lambda_{A}$ and $\lambda_{M}$ be the largest eigenvalue of $\left(I+B_{k}\right)^{T}\left(I+B_{k}\right) \quad(k=1,2, \cdots), A+A^{T}$, $M(y, x)+M^{T}(y, x)$ respectively, if there exists a constant $\alpha>1$ such that $\ln \left(\alpha \beta_{k}\right)+\left(\lambda_{A}+\lambda_{M}\right) \tau_{k} \leq 0, \quad k=1,2, \cdots$, then system (4) and (2) are global Asymptotic synchronization. Here $\tau_{k}=t_{k}-t_{k-1}$ is pulse interval.

Proof: Choose the Lyapunov function $V(e)=e^{T} e$, then for $t \in\left(t_{k-1}, t_{k}\right],(k=1,2, \cdots)$ we have

$$
\begin{aligned}
\dot{V}(e) & =\dot{e}^{T} e+e^{T} \dot{e}=[A e+M(y, x) e]^{T} e+e^{T}[A e+M(y, x) e] \\
& =e^{T}\left[A+A^{T}\right] e+e^{T}\left[M(y, x)+M^{T}(y, x)\right] e \\
& \leq\left(\lambda_{A}+\lambda_{M}\right) e^{T} e=\left(\lambda_{A}+\lambda_{M}\right) V(e)
\end{aligned}
$$

So

$$
V(e(t)) \leq V\left(e\left(t_{k-1}^{+}\right)\right) \exp \left[\left(\lambda_{A}+\lambda_{M}\right) \cdot\left(t-t_{k-1}\right)\right]
$$

On the other hand, when $t=t_{k},(k=1,2, \cdots)$, we get

$$
\begin{aligned}
V\left(e\left(t_{k}^{+}\right)\right) & =\left[\left(I+B_{k}\right) e\left(t_{k}\right)\right]^{T}\left(I+B_{k}\right) e\left(t_{k}\right) \\
& =e^{T}\left(t_{k}\right)\left(I+B_{k}\right)^{T}\left(I+B_{k}\right) e\left(t_{k}\right) \\
& \leq \beta_{k} V\left(e\left(t_{k}\right)\right)
\end{aligned}
$$

According to the inequality (6) and (7), we can get

$$
\begin{aligned}
V(e(t)) \leq & V\left(e\left(t_{0}\right)\right) \beta_{1} \beta_{2} \cdots \beta_{k} \exp \left[\left(\lambda_{A}+\lambda_{M}\right)\left(t-t_{0}\right)\right] \\
= & V\left(e\left(t_{0}\right)\right)\left\{\beta_{1} \exp \left[\left(\lambda_{A}+\lambda_{M}\right) \tau_{1}\right]\right\} \\
& \left\{\beta_{2} \exp \left[\left(\lambda_{A}+\lambda_{M}\right) \tau_{2}\right]\right\} \cdots \\
& \left\{\beta_{k} \exp \left[\left(\lambda_{A}+\lambda_{M}\right)\left(t-t_{k}\right)\right]\right\} \\
\leq & V\left(e\left(t_{0}\right)\right) \beta_{k} \exp \left[\left(\lambda_{A}+\lambda_{M}\right)\left(t-t_{k}\right)\right] / \alpha^{k-1}
\end{aligned}
$$

Considering $V\left(e\left(t_{0}\right)\right)$ and $\exp \left[\left(\lambda_{A}+\lambda_{M}\right)\left(t-t_{k}\right)\right] \frac{1}{2}$ are both bounded, so $\lim V(e)=0$. Then the trivial solution of system (5) is global asymptotically stable. That is, system (4) and (2) are global asymptotic synchronization.

Now, according matrix theory, we have

$\lambda_{M} \leq\left\|M(y, x)+M^{T}(y, x)\right\|$, where $\|\cdot\|$ denotes any kinds of norms of a matrix. We can obtain the following corollary:

Corollary: if there exists a constant $\alpha>1$ such that

$$
\begin{aligned}
& \ln \left(\alpha \beta_{k}\right)+\left(\lambda_{A}+\left\|M(y, x)+M^{T}(y, x)\right\|\right) \tau_{k} \leq 0, \\
& k=1,2, \cdots
\end{aligned}
$$

then system (4) and (2) are global asymptotic synchronization.

\section{Numerical Simulation}

In this section, we present some numerical simulations to demonstrate our results. We choose the parameters of Lorenz system (1) as $\sigma=10, \rho=28, \beta=8 / 3$, then we can calculate that $\lambda_{A}=28.05$. By observing the Lorenz attractor (Figure 1), we can get state variable's value ranges: $-20 \leq x_{1} \leq 20,0 \leq x_{2} \leq 50,-20 \leq x_{3} \leq 20$, and work out $\lambda_{M} \leq 140$. Take the equal impulsive intervals and the equal impulsive feedback gain matrix, that is, set $\tau_{k}=\tau, \quad B_{k}=B=\operatorname{diag}(\mu, \mu, \mu), k=1,2, \cdots$, then $\beta_{k}=(\mu+1)^{2}$. According to the corollary in the previous section, if $0<\tau \leq-\left[\ln \alpha+\ln (\mu+1)^{2}\right] / 168.05$, then system (4) and (2) are global asymptotically synchronization. Let $\mu=-0.5, \alpha=1.1$, then $0<\tau \leq 0.0077$. Figure 2 shows the simulation results in which initial

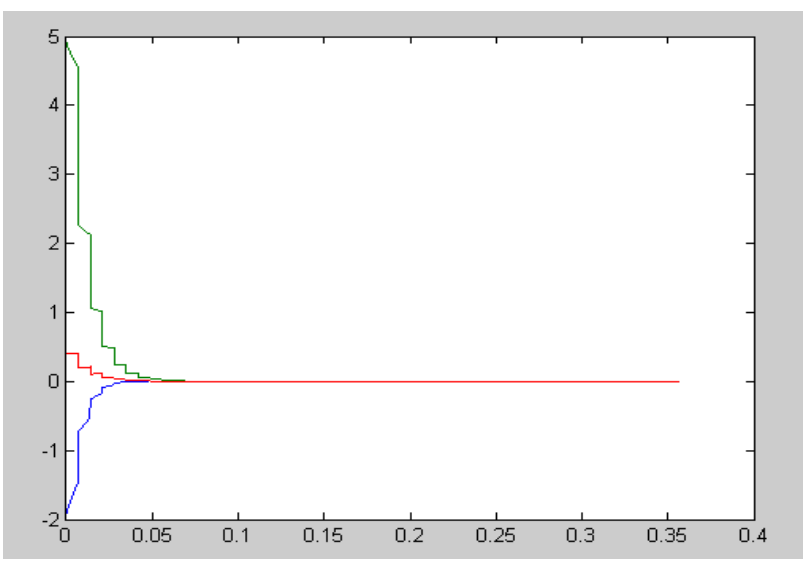

Figure 2. Synchronization error of Lorenz chaotic system. 
value of drive system (2) is $(2,0.1,0.1)^{T}$ while initial value of response system (4) is $(0.1,5,0.5)^{T}$, and $\mu=-0.5$, $\tau=0.007$. We can see that synchronization error converges to zero quickly.

\section{Acknowledgements}

This work is supported jointly by the Natural Sciences Foundation of China under Grant No.60963025, Natural Sciences Foundation of Hainan Province under Grant No. 110007 and the Start-up fund of Hainan Normal University under Project No. 00203020201.

\section{REFERENCES}

[1] Pecora L. and Carroll T., "Synchronization in chaotic systems,” Physical Review Letters, 1990, 64 (8) 821-824.

[2] Hasler M., "Synchronization of chaotic systems and transmission of information," Int. J. Bifurcation and Chaos, 1998, 8 (4) 647-659.

[3] Lu J.Q., Daniel W.C. and Cao J.D., "A unified synchronization criterion for impulsive dynamical networks," Automatica 2010, 46 (7) 1215-1221.

[4] Sun J.T. and Zhang Y.P., "Impulsive control and synchronization of Chua's oscillators," Mathematics and
Computer in Simulation, 2004, 66(6) 499-508.

[5] Zhang L.P. and Jiang H.B., "Impulsive generalized synchronization for a class of nonlinear discrete chaotic systems," Communications in Nonlinear Science and $\mathrm{Nu}$ merical Simulation, 2011, 16(4) 2027-2032.

[6] Zhang R., Xu Z.Y., Yang S.X. and He X.M., "Generalized synchronization via impulsive control," Chaos Solition Fract, 2008, 38(1) 97-105.

[7] Zhang L.P., Jiang H.B. and Bi Q.S., "Reliable impulsive lag synchronization for a class of nonlinear discrete chaotic systems,” Nonlinear Dynam, 2010, 59 (4) 529-534.

[8] Xu X.H., Cao Y. and Zhang J.Y., "Global Exponential Synchronization of Impulsive Chaotic Neural Network," Journal of Southwest Jiaotong University, 2009, 44 (6) 887-892

[9] Sun J.T. and Zhang Y.P., "Impulsive control of Rössler systems,” Physics Letters, 2003, 306 (5) 306-312.

[10] Wang Y.W., Guan Z.H. and Xiao J.W., "Impulsive control for synchronization of a class of continuous systems," Chaos solition fractals, 2004, 14 (1) 199-203.

[11] Tang G. N., Luo X. S. and Kong L. J., "Study on impulsive control of chaotic Lorenz system," Journal of guangxi normal university, 2000, 18 (1) 13-16. 